\title{
Enhancement and Narrowing of Excitonic Lines in AlInN/GaN Heterostructures
}

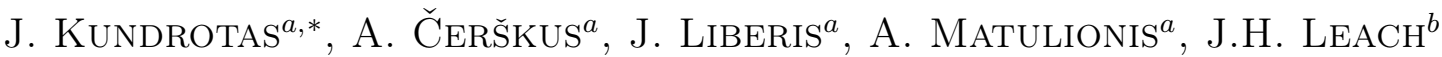 \\ AND A.H. MORKOÇ \\ ${ }^{a}$ Semiconductor Physics Institute, Center for Physical Sciences and Technology \\ A. Goštauto 11, LT-01108 Vilnius, Lithuania \\ ${ }^{b}$ Department of Electrical and Computer Engineering, Virginia Commonwealth University \\ Richmond, Virginia, 23284 USA
}

\begin{abstract}
A study of the photoluminescence properties of AlInN/GaN in comparison with the spectrum of the GaN active layer of the same heterostructure is presented. The strong intensity lines of the observed photoluminescence spectra are associated with the formation, enhancement and narrowing of the excitonic lines in the flat band region of the active GaN layer. The phenomena in the presence of electric field near the heterostructure interface with the two-dimensional electron system are associated with nonlinear behaviour of recombination processes.
\end{abstract}

PACS: 78.55.-m, 78.66.-w, 71.55.Eq, 71.35.-y

\section{Introduction}

Nitride heterostructures $\mathrm{AlGaN}(\operatorname{InAlN}) / \mathrm{GaN}$ with two-dimensional channels are widely used in high performance electronic devices [1], in particular for developing heterostructure field effect transistors (HFETs) [2]. Investigation of photoluminescence (PL) spectra and other optical properties are crucial for understanding of intrinsic properties of the heterostructures important for advancing the nitride technology.

It is still unclear what the origin of the optical transitions is vis a vis the recombination processes in nominally undoped or selectively doped heterostructures based on compound semiconductors in general. The radiative recombination of photoexcited carriers is affected by the strong internal electric field formed at the heterointerface. The field is of special importance for the photoluminescence of bimolecular excitons formed by electrons and holes excited by incident photons. For example, the ensuing recombination induces superlinearities in the transient PL spectra of $\mathrm{Al}_{x} \mathrm{Ga}_{1-x} \mathrm{As} / \mathrm{GaAs}$ heterostructures recorded during the pump-probe experiment [3]: an enhancement of the PL intensity was also observed $[3,4]$. So far, only a few similar optical studies have been reported for GaN heterostructures [5-9], and therefore, the topic requires more investigations. In this vein, our investigations show both enhancement and narrowing of the excitonic lines in an AlInN/GaN heterostructure that confines a two-dimensional electron system near

\footnotetext{
* corresponding author; e-mail: kundrot@pfi.lt
}

the heterointerface. In the present work, the PL spectra of the AlInN/GaN heterostructures have been compared with the spectra of the active GaN layers of the same structure. A possible model of the exciton photoluminescence enhancement in AlInN/GaN heterostructures is discussed. The influences of the built-in electric field and several recombination mechanisms are also considered.

\section{Samples and measurement technique}

The AlInN/GaN structures were grown on (0001) sapphire substrates in a vertical low-pressure metal-organic chemical vapor deposition system [10]. The growth was initiated with a 250-300 nm AlN buffer layer followed by an approximately $d_{\text {active }}=4 \mu \mathrm{m} \mathrm{GaN}$ active layer. After depositing an AlN spacer layer ( $d_{\mathrm{sp}}$ thickness), a layer of $\mathrm{Al}_{1-x} \operatorname{In}_{x} \mathrm{~N}$ (thickness $d$ ) was grown, and finally capped with GaN layer $\left(d_{\text {cap }}\right)$. The AlInN/GaN structure contains a two-dimensional electron gas (2DEG) in the active layer of GaN near the interface. The parameters and the measured characteristics of the samples are shown in Table.

The samples were excited with a HeCd laser $(15 \mathrm{~mW})$ operating in a continuous wave mode with photon energy of $3.81 \mathrm{eV}(\lambda=325 \mathrm{~nm})$. The photoluminescence signal was dispersed by a monochromator and detected by a thermoelectrically cooled GaAs photomultiplier operating in the photon counting regime. A closed cycle helium optical cryostat enabled sample temperatures from ambient $300 \mathrm{~K}$ down to $3.6 \mathrm{~K}$.

The conventional measurements of PL spectra were made for polarizations of $\alpha(\boldsymbol{k} \| \boldsymbol{c}, \boldsymbol{E} \perp \boldsymbol{c})$, where $\boldsymbol{k}$ and 
$\boldsymbol{E}$ are the wave vector and electric field of the emitted light, and $c$ is the principal crystallographic axis. The heterostructures were illuminated with the laser through the cap layers. For further investigation the GaN active layers, the GaN layer was also illuminated through the sapphire substrate from the backside of the heterostructure.

Sample parameters and summary of measured characteristics:

$\mu_{\text {Hall }}$ is 2DEG Hall mobility and $N_{\mathrm{s}}$ is 2DEG sheet density in GaN active layer, respectively.

\begin{tabular}{c|c|c|c|c|c|c|c}
\hline \hline \multirow{3}{*}{ Sample } & GaN & \multicolumn{2}{|c|}{$\mathrm{Al}_{1-x} \operatorname{In}_{x} \mathrm{~N}$} & $\mathrm{AlN}$ & $\mathrm{GaN}$ & \multicolumn{2}{c|}{$2 \mathrm{DEG}, 300 \mathrm{~K}$} \\
\cline { 2 - 8 } & $\begin{array}{c}d_{\text {cap }} \\
{[\mathrm{nm}]}\end{array}$ & $\begin{array}{c}d \\
{[\mathrm{~nm}]}\end{array}$ & $x$ & $\begin{array}{c}d_{\mathrm{sp}} \\
{[\mathrm{nm}]}\end{array}$ & $\begin{array}{c}d_{\text {active }} \\
{[\mu \mathrm{m}]}\end{array}$ & $\begin{array}{c}\mu_{\text {Hall }} \\
{\left[\mathrm{cm}^{2} /(\mathrm{V} \mathrm{s})\right]}\end{array}$ & $\begin{array}{c}N_{\mathrm{s}} \times 10^{13} \\
{\left[\mathrm{~cm}^{-2}\right]}\end{array}$ \\
\hline CVD2081 & 1 & $17-20$ & 0.18 & 0.3 & 4 & 400 & 1.4 \\
CVD2100 & 1 & $17-20$ & 0.15 & 1 & 4 & 1600 & 1 \\
CVD2383 & $1-2$ & $\approx 25$ & 0.14 & $0.8-1$ & 4 & $\approx 1200$ & $\approx 1$ \\
CVD2424 & 2 & $21.5-25$ & $0.17-0.18$ & $0.8-1$ & 4 & $\approx 1060$ & $\approx 2.7$
\end{tabular}

\section{Experimental results and discussion}

The PL spectra of the AlInN/GaN heterostructure (\#CVD2100) and its GaN active layer at $3.6 \mathrm{~K}$ are presented in Fig. 1. The spectrum of the active GaN layer has only one broad band near the forbidden energy gap with a full width at half maximum (FWHM) that equals $16 \mathrm{meV}$. The spectrum of the AlInN/GaN heterostructure contains well resolved three GaN-related $X_{A}, X_{B}$ and $X_{C}$ excitonic transitions and also a weak peak $X_{A}(n=2)$, which we attribute to an excited state of the $X_{A}$ exciton. The FWHM of the excitonic line $X_{A}$ is $5 \mathrm{meV}, X_{B}$ is $3.7 \mathrm{meV}$ and $X_{C}$ is $3.4 \mathrm{meV}$, respectively. We also established that the exciton line $X_{A}$ of the heterostructure is about 15 times more intense than the PL maximum of the GaN active layer. From Fig. 1 we can summarize that the PL spectrum of the GaN layer is broadband in nature, while the spectrum of the heterostructure splits into narrow individual excitonic lines. The investigated heterostructures demonstrated two effects: the enhancement of the excitonic photoluminescence and the narrowing of the excitonic lines in comparison to the PL spectrum of the GaN active layer of the same structure.

The residual strain influences the spectrum of excitonic transitions [11-15], and, in our case, the spectrum is shifted by approximately $10 \mathrm{meV}$ towards the higher energy region compared to the spectrum of a fully relaxed GaN layer $[1,16,17]$. The emission band at $3.55 \mathrm{eV}$ is attributed to the emission from AlInN layer possibly associated with very large Stokes shifts of $0.4-0.8 \mathrm{eV}$ observed near a composition range of $0.18[18,19]$. The origin of this effect is not clear. It has been proposed that such a large energy difference between the band edge and the luminescence could be ascribed to the composition fluctuations of In or the presence of deep defects or impurities acting as preferential recombination centers [18].

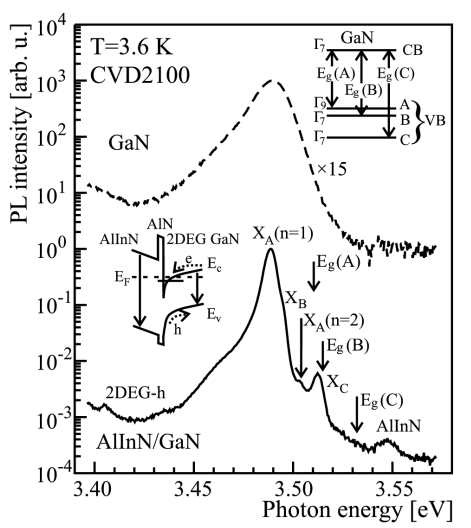

Fig. 1. The PL spectra of the AlInN/GaN heterostructure (bottom curve) and its GaN active layer (top curve) at $3.6 \mathrm{~K}$. For the sake of clarity, the top curve is shifted vertically and multiplied with 15 .

The 2DEG is induced by "spontaneous and piezoelectric polarization" in the investigated AlInN/GaN heterostructures, but the source of free electrons is of different origin. The positive polarization charge at the interface is responsible for the tendency to collect the free electrons from other sources [20]. The sources of free electrons can be either surface states [20-23] and/or the AlInN layer [18]. For example, a typical AlInN layer contains a free electron concentration of $(1-5) \times 10^{18} \mathrm{~cm}^{-3}$, possibly due to nitrogen vacancies acting as shallow donors or residual oxygen impurities [18]. The emission at $3.41 \mathrm{eV}$ can be related to the 2DEG system, whose emission spectral peak position varies in the region of 3.38-3.44 eV [8].

The PL spectra of various AlInN/GaN heterostructures and its GaN active layers at $3.6 \mathrm{~K}$ are shown in Fig. 2. While the spectra of GaN active layer spectra 


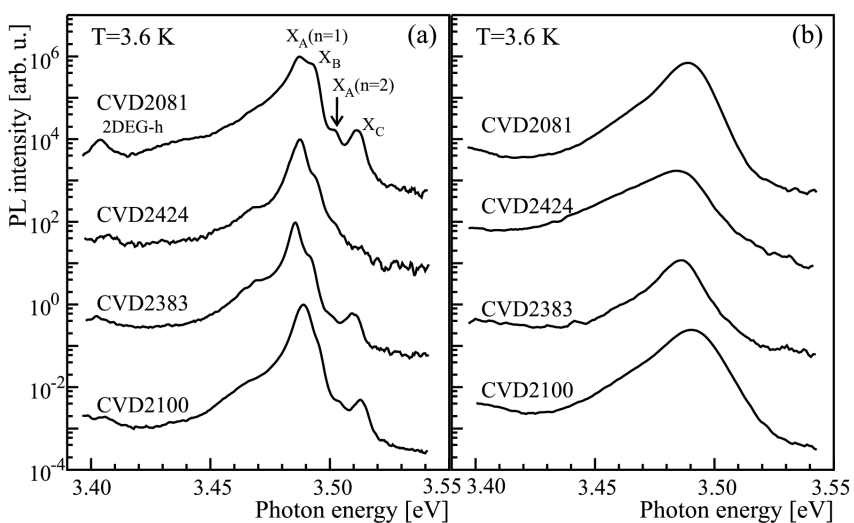

Fig. 2. The PL spectra of various AlInN/GaN heterostructures (a) and its GaN active layers (b) at $3.6 \mathrm{~K}$. For the sake of clarity, the curves are shifted vertically.

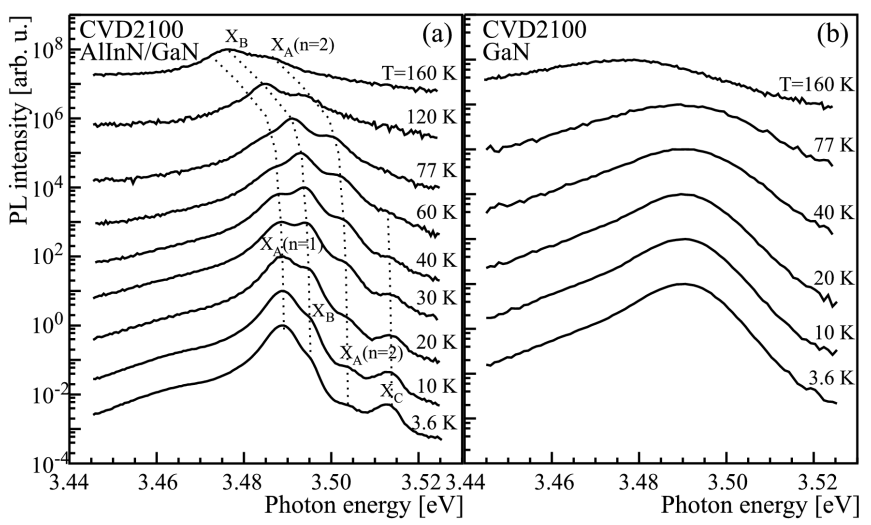

Fig. 3. The PL spectra of the AlInN/GaN heterostructure (a) and its GaN active layer (b) at different temperatures.

are broad (the FWHM varies from $12.8 \mathrm{eV}$ to $22 \mathrm{meV}$ for different samples, Fig. 2b), the spectra of the AlInN/ GaN heterostructures for all samples show narrowed and similar structure (Fig. 2a). Thus, the heterostructure spectra demonstrate features absent for the GaN active layer. In particular, the free exciton transitions $X_{A}, X_{B}$, and $X_{C}$, observed in all the PL spectra, are the same as those recorded for good quality GaN layers [24-26]. In other words, the spectrum of a heterostructure reflects fundamental optical properties of GaN.

In GaN of hexagonal symmetry, the $A$ and $B$ transitions dominate for the light polarization $\boldsymbol{E}$ being perpendicular to the $\boldsymbol{c}$ axis. However, the $C$ exciton is mostly active for the parallel polarization ( $\boldsymbol{E}$ parallel to the $c$ axis), but small contributions come from perpendicular components [27-30]. This non-zero contribution adds up and gives finite transition for $C$ exciton in PL for $\alpha$-polarization. Moreover, the relative intensities depend on the electric fields and residual stresses $[12,15]$.

The PL spectra of the AlInN/GaN heterostructure (\#CVD2100) and its GaN active layer at different tem- peratures are shown in Fig. 3. It is clear that the excitonic structure of the heterostructure (Fig. 3a) survives in the entire investigated temperature range of 3.6-160 K.

The origin of the excitonic PL in the heterostructures with the 2DEG is not as evident, as it may seem at a first glance. The proposed effect of the built-in electric field may explain the origin of the excitonic photoluminescence in $\mathrm{Al}_{x} \mathrm{Ga}_{1-x} \mathrm{As} / \mathrm{GaAs}$ heterostructures with quasi-triangular quantum wells at the heterointerface [4, 31]. Similar conditions can be found in the investigated nitride heterostructure, and the excitonic luminescence can be discussed from the same point of view.

The incident laser beam excites electrons within a certain light penetration depth. These photoexcited carriers can form excitons in the GaN active layer and may then radiatively recombine which results in photon emission. However, the presence of a strong built-in electric field excludes exciton formation in the close proximity of the heterointerface region.

At the interface, the 2DEG occupies the triangular potential well and induces an electric field at the order of 1 $\mathrm{MV} / \mathrm{cm}$. However, an electric field above $100 \mathrm{kV} / \mathrm{cm}$ can destroy excitons by tunneling [32]. Therefore, the electron-hole pairs near the interface are dissolved by the electric field and will either move towards (electrons) or away (holes) from the interface. During this process the dissolved electron and hole cannot meet each other, but may have the possibility to catch holes and electrons originating from other pairs through bimolecular formation of exciton. The resulting process can lead to an accumulation of free carriers that increases the number of excitons in the GaN layer at the edge of the 2DEG channel where the "flat band" condition is satisfied. Theoretical analysis [33] shows essential nonlinearity of the excitonic PL intensity when impurities take part in the recombination process. The nonlinear dependence of excitonic PL intensity on carrier density may explain the enhancement of excitonic lines. Recently, similar changes of the excitonic PL spectra have been reported for AlGaAs/GaAs heterostructures [34].

The origin of the PL excitonic line-narrowing phenomenon is not yet clear. It is known that the excited electrons and holes in epitaxial layers remain mainly near residual donors, acceptors or other inhomogeneities that are randomly distributed in space. This interaction causes inhomogeneous broadening of the excitonic linewidth. The FWHM of the excitonic lines at low temperatures in AlInN/GaN heterostructures are similar to the linewidths of a high quality GaN crystal, thus suggesting that the interaction of excitons with crystal imperfections is affected by the heterostructure. The light-induced narrowing of excitonic absorption lines in GaN was observed in Ref. [35]. The effect is most likely due to the reduction of the strength of the electric field around the negatively charged dislocations after the sample has been illuminated. Changes in PL spectra were not observed when the illumination intensity was changed. 


\section{Summary}

We have investigated the PL spectra for AlInN/ GaN heterostructures containing 2DEG near the interface. Strong photoluminescence intensities were associated with the formation of free excitons in a flat band region of the GaN active layer. We also observed an enhancement of the exitonic PL intensity and a narrowing of the excitonic lines. This phenomenon may be related to the nonlinear behavior of the excitonic PL in the AlInN/GaN heterostructures.

\section{Acknowledgments}

This work is supported, in part, by grants FA9550-04-1-04-14 and FA8655-09-1-3103 from the US Air Force Office of Scientific Research and the US European Office of Aerospace Research and Development under the direction of Dr. Kitt Reinhardt and Dr. Scott Dudley.

\section{References}

[1] H. Morkoç, Handbook of Nitride Semiconductors and Devices, Vols. I-III, Wiley-VCH, Weinheim 2008-9.

[2] J. Liberis, I. Matulionienienè, A. Matulionis, E. Šermukšnis, J. Xie, J.H. Leach, H. Morkoç, Phys. Status Solidi A 206, 1385 (2009).

[3] J.X. Shen, R. Pittini, Y. Oka, Phys. Status Solidi B 221, 545 (2000).

[4] J.X. Shen, R. Pittini, Y. Oka, Phys. Rev. B 64, 195321 (2001).

[5] J.P. Bergman, T. Lundström, B. Monemar, H. Amano, I. Akasaki, Appl. Phys. Lett. 69, 3456 (1996).

[6] B. Shen, T. Someya, O. Moriwaki, Y. Arakawa, Appl. Phys. Lett. 76, 679 (2000).

[7] H.K. Kwon, C.J. Eiting, D.J.H. Lambert, B.S. Shelton, M.M. Wong, T.G. Zhu, R.D. Dupuis, J. Appl. Phys. 90, 1817 (2001).

[8] K.B. Nam, J. Li, M.L. Nakarmi, J.Y. Lin, H.X. Jiang, Appl. Phys. Lett. 81, 1809 (2002).

[9] H.-S. Kwack, Y.-H. Cho, G.H. Kim, M.R. Park, D.H. Youn, S.B. Bae, K.-S. Lee, J.-H. Lee, J.-H. Lee, T.W. Kim, T.W. Kang, K.L. Wang, Appl. Phys. Lett. 87, 041909 (2005)

[10] J. Xie, X. Ni, Mo Wu, J.H. Leach, Ü. Özgür, H. Morkoç, Appl. Phys. Lett. 91, 132116 (2007).

[11] B. Gil, O. Briot, R.-L. Aulombard, Phys. Rev. B 52, R17028 (1995).

[12] B. Gil, F. Hamdani, H. Morkoç, Phys. Rev. B 54, 7678 (1996).

[13] B. Gil, O. Briot, Phys. Rev. B 55, 2530 (1997).

[14] A.A. Yamaguchi, Y. Mochizuki, H. Sunakawa, A. Usui, J. Appl. Phys. 83, 4542 (1998).

[15] O. Aoudé, P. Disseix, J. Leymarie, A. Vasson, M. Leroux, E. Aujol, B. Beaumont, A. Trassoudaine, Y. André, Phys. Rev. B 77, 045206 (2008).

[16] R. Stępniewski, K.P. Korona, A. Wysmołek, J.M. Baranowski, K. Pakuła, M. Potemski, G. Martinez, I. Grzegory, S. Porowski, Phys. Rev. B 56, 15151 (1997).
[17] S.W. Lee, J.-S. Ha, H.-J. Lee, H.-J. Lee, H. Goto, T. Hanada, T. Goto, K. Fujii, M.W. Cho, T. Yao, J. Phys. D, Appl. Phys. 43, 175101 (2010).

[18] R. Butté, J.-F. Carlin, E. Feltin, M. Gonschorek, S. Nicolay, G. Christmann, D. Simeonov, A. Castiglia, J. Dorsaz, H.J. Buehlmann, S. Christopoulos, G. Baldassarri Höger von Högersthal, A.J.D. Grundy, M. Mosca, C. Pinquier, M.A. Py, F. Demangeot, J. Frandon, P.G. Lagoudakis, J.J. Baumberg, N. Grandjean, J. Phys. D, Appl. Phys. 40, 6328 (2007).

[19] K. Wang, R.W. Martin, D. Amabile, P.R. Edwards, S. Hernandez, E. Nogales, K.P. O'Donnell, K. Lorenz, E. Alves, V. Matias, A. Vantomme, D. Wolverson, I.M. Watson, J. Appl. Phys. 103, 073510 (2008).

[20] J.P. Ibbetson, P.T. Fini, K.D. Ness, S.P. DenBaars, J.S. Speck, U.K. Mishra, Appl. Phys. Lett. 77, 250 (2000).

[21] G. Koley, M.G. Spencer, Appl. Phys. Lett. 86, 042107 (2005).

[22] Z.H. Wu, M. Stevens, F.A. Ponce, W. Lee, J.H. Ryou, D. Yoo, R.D. Dupuis, Appl. Phys. Lett. 90, 032101 (2007).

[23] M. Gonschorek, J.-F. Carlin, E. Feltin, M.A. Py, N. Grandjean, V. Darakchieva, B. Monemar, M. Lorenz, G. Ramm, J. Appl. Phys. 103, 093714 (2008).

[24] D. Volm, K. Oettinger, T. Streibl, D. Kovalev, M. Ben-Chorin, J. Diener, B.K. Meyer, J. Majewski, L. Eckey, A. Hoffmann, H. Amano, I. Akasaki, K. Hiramatsu, T. Detchprohm, Phys. Rev. B 53, 16543 (1996).

[25] G. Pozina, J.P. Bergman, T. Paskova, B. Monemar, Appl. Phys. Lett. 75, 4124 (1999).

[26] T.V. Shubina, T. Paskova, A.A. Toropov, S.V. Ivanov, B. Monemar, Phys. Rev. B 65, 075212 (2002).

[27] R. Dingle, D.D. Sell, S.E. Stokowski, M. Ilegems, Phys. Rev. B 4, 1211 (1971).

[28] G.D. Chen, M. Smith, J.Y. Lin, H.X. Jiang, S.-H. Wei, M. Asif Khan, C.J. Sun, Appl. Phys. Lett. 68, 2784 (1996).

[29] K. Torii, T. Deguchi, T. Sota, K. Suzuki, S. Chichibu, S. Nakamura, Phys. Rev. B 60, 4723 (1999).

[30] P.P. Paskov, T. Paskova, P.O. Holtz, B. Monemar, Phys. Status Solidi A 201, 678 (2004).

[31] B.M. Ashkinadze, E. Linder, V. Umansky, Phys. Rev. B 62, 10310 (2000).

[32] B. Monemar, P.P. Paskov, J.P. Bergman, A.A. Toropov, T.V. Shubina, T. Malinauskas, A. Usui, Phys. Status Solidi B 245, 1723 (2008).

[33] T. Schmidt, K. Lischka, W. Zulehner, Phys. Rev. B 45, 8989 (1992).

[34] J. Kundrotas, A. Čerškus, V. Nargelienè, A. Sužiedèlis, S. Ašmontas, J. Gradauskas, A. Johannessen, E. Johannessen, V. Umansky, J. Appl. Phys. 108, 063522 (2010).

[35] P. Trautman, K. Pakula, R. Bożek, J.M. Baranowski, Appl. Phys. Lett. 83, 3510 (2003). 